\title{
Magnetization and correlation length of the easy-plane ferromagnetic chain: Numerical analysis and application to $\left(\mathrm{C}_{6} \mathrm{H}_{11} \mathrm{NH}_{3}\right) \mathrm{CuBr}_{3}$
}

\author{
G. Kamieniarz, ${ }^{*}$ F. Mallezie, and R. Dekeyser \\ Instituut voor Theoretische Fysica, Universiteit Leuven, B-3030 Leuven, Belgium
}

(Received 4 April 1988)

\begin{abstract}
The ferromagnetic spin- $\frac{1}{2}$ one-dimensional $X X Z$ Heisenberg model is studied numerically by quantum Monte Carlo analysis and extrapolations in $1 / N$ of finite-size chain data. Reliable magnetization and correlation-length estimates are presented and a quantitative agreement with recent experimental data on the compound $\left(\mathrm{C}_{6} \mathrm{H}_{11} \mathrm{NH}_{3}\right) \mathrm{CuBr}_{3}$ is found.
\end{abstract}

\section{INTRODUCTION}

In recent years one-dimensional (1D) magnetic systems attracted a great deal of theoretical and experimental interest. $^{1-10} \mathrm{~A}$ well-known example is $\left(\mathrm{C}_{6} \mathrm{H}_{11} \mathrm{NH}_{3}\right) \mathrm{CuBr}_{3}$ (CHAB) (Refs. 5 and 6), which displays a magnetic order below $T_{c}=1.5 \mathrm{~K}$. The intrachain interactions are predominant, and respective interchain interactions are smaller by a few orders of magnitude. The latter are responsible for a three-dimensional ordering appearing below $T_{c}$. Some properties of CHAB have been interpret$\mathrm{ed}^{6,11}$ through the one-dimensional spin- $\frac{1}{2}$ Heisenberg model with the ferromagnetic nearest-neighbor interaction of about $55 \mathrm{~K}$, containing also $5 \%$ easy-plane anisotropy.

In the present analysis we consider the anisotropic Heisenberg Hamiltonian

$$
\begin{aligned}
\hat{H}= & -2 J \sum_{i}\left(S_{i}^{x} S_{i+1}^{x}+S_{i}^{y} S_{i+1}^{y}+\Delta S_{i}^{z} S_{i+1}^{z}\right) \\
& -g \mu_{B} B \sum_{i} S_{i}^{x},
\end{aligned}
$$

where

$$
J / k_{B}=55 \pm 5 \mathrm{~K}, \Delta=0.95, \quad g=2.01 \pm 0.02
$$

with no adjustable parameters and $S=\frac{1}{2}$. The interest in CHAB follows from the fact that for the Hamiltonian (1) the nonlinear excitations should not be negligible. CHAB thus constitutes an example of the compound where magnetic solitons should show up. In the presence of a symmetry-breaking field applied above $T_{c}$ within the easy plane (assumed to be the $X Y$ plane), the corresponding equation of motion of the spin can be mapped onto a sine-Gordon (SG) equation ${ }^{12}$ provided that (a) the continuum limit is considered, (b) the classical limit is taken, and (c) anisotropy is large enough to confine the spins within the easy plane. It turns out that the effect of the various approximations yielding the mapping of the original quantum Hamiltonian onto the SG counterpart is unknown and the deviations (reported in more detail before $^{13}$ ) of the corresponding SG results from the experimental observations go beyond the acceptable rate. Thus it has become important to interpret the experimental data by having recourse to the quantum Hamiltonian (1).
A new complementary type of theoretical analysis is based on numerical computations. Quantum Monte Carlo (QMC) and transfer matrix (QTM) techniques ${ }^{14}$ are related to the Trotter formalism ${ }^{15}$ whereas finite-size calculations ${ }^{13}$ refer to an extrapolation technique. According to the Trotter formula, ${ }^{15}$ the one-dimensional quantum Hamiltonian is mapped onto a corresponding classical Hamiltonian on a strip. The length $N$ of the chains accessible in the QMC and QTM calculations is of the or$\operatorname{der} N=100$. The width $m$ of the strip in the Trotter direction is usually limited to $m=8$. The length $N$ of the chains considered in the finite-size calculations is of the order $N=10$; nevertheless, the corresponding results extrapolated to infinite $N$ have been rather well established since the pioneering work of Bonner and Fisher. ${ }^{16}$ For the isotropic Heisenberg model and the $X Y$ model, the progression of QMC (Ref. 17) and QTM (Ref. 18) results towards those of the finite-size calculations has been demonstrated.

A number of experiments have been performed on CHAB: magnetization measurements, ${ }^{5,11}$ ferromagnetic resonance experiments, ${ }^{19}$ specific-heat measurements without $^{5}$ and in the presence of a magnetic field ${ }^{6,20}$ as well as neutron-scattering experiments. ${ }^{21}$ The specificheat data can be analyzed in terms of the excess specific heat $\Delta C(B, T)=C(B, T)-C(0, T)$ as a function of $T / \sqrt{B}$. The excess specific heat displays the peak features. $^{20} \mathrm{Up}$ until now a satisfactory quantitative agreement between experiment and theoretical calculations has not been realized as far as the peak heights and the peak positions in the specific heat are concerned. However, in contrast to the QMC and QTM technique, ${ }^{14}$ the finite-size analysis ${ }^{13}$ revealed a quantitative agreement for the peak heights. Moreover, an extension of the finite-size calculations ${ }^{22}$ leads to a behavior of the correlation length consistent with that found experimentally. ${ }^{21}$

Here we supplement the previous analysis ${ }^{22}$ of the correlation length with the QMC simulations and we present new magnetization data (partially reported in Ref. 23) calculated for the magnetic field applied in the easy plane. Our results are consistent with those found before $\mathrm{e}^{21,22}$ as far as the correlation length is concerned and they fit the experimental magnetization data within the error bars. 
The article is organized as follows. In Sec. II a brief description of the finite-size analysis and the corresponding results are presented. In Sec. III some details about the QMC technique are described and the corresponding data are plotted. Contact with some exact theoretical results is also made. The paper ends with concluding remarks in Sec. IV.

\section{FINITE-SIZE MAGNETIZATION DATA}

Here we report on our numerical finite-size analyses of the magnetization for the Hamiltonian (1) with the field in the easy plane and parameters quoted in Eq. (2). Our calculations proceed as follows. The matrix representation of the Hamiltonian (1) in the $x$ representation is diagonalized numerically for finite chains with size $N$ up to 10 and free boundary conditions. The magnetization of a given chain is expressed in terms of the eigenvalues $E_{\alpha}$ and the corresponding eigenvectors $|\alpha\rangle$ and is evaluated from the formula

$$
M^{\text {mole }}=g \mu_{B} \sigma R / k_{B} \equiv \sigma M_{S},
$$

where

$$
\sigma=\frac{1}{N}\left(\sum_{\alpha}\left\langle\alpha\left|\sum_{i} S_{i}^{x}\right| \alpha\right\rangle e^{-\beta E_{\alpha}}\right) / \sum_{\alpha} e^{-\beta E_{\alpha}}
$$

denotes the relative magnetization per site whereas $\mu_{B}, R, k_{B}$ are standard constants. The numerical data for different values of the size $N$ are then analyzed in terms of the inverse length $1 / N$ and extrapolated. Linear and parabolic fitting in $1 / N$ is performed and data from $N=6$ up to $N=10$ are taken into account. The number of points involved in the extrapolation procedure is denoted by $n$. This number runs over the set $2 \leq n \leq 5$ for the linear fitting. The value $n=2$ stands for the linear fitting based on the data referring to $N=10$ and $N=9$. All the data $(N \geq 6)$ are taken into account for $n=5$. For the parabolic fitting $3 \leq n \leq 5$. When $n$ is changed from its maximal value towards the minimal one the extrapolated data display an increasing or decreasing tendency. If the data for both the linear and the parabolic fitting increase with decreasing $n$, we report the maximal extrapolated value. If the decreasing tendency is found for both fitting procedures, the lowest from the extrapolated data is reported. As an error estimate we assume the corresponding difference between the results of the linear and parabolic fitting. Sometimes a third possibility is found: the larger values for one fitting display a progression in the downward direction whereas the lower values for the other fitting progress in the upward direction. These convergent data are reported by intercepts. The case of a divergent tendency has not been found in our analysis.

We skip other details about the extrapolation procedure as it was rather extensively demonstrated previously. ${ }^{13,22}$ We proceed now to present our results. The molar magnetization (3) versus the applied field $B$ in $\mathrm{kG}$ is plotted in Fig. 1 for temperatures $T=4.2,6$, and $10 \mathrm{~K}$. The curves represent the experimental data. ${ }^{11}$ Our results are reported by open squares or by intercepts. The estimated error bars are attached to the squares if they

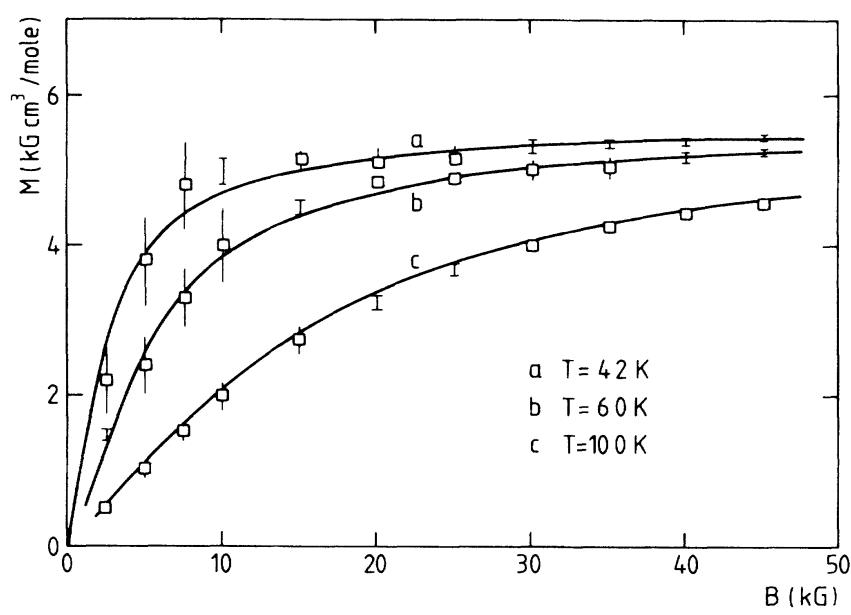

FIG. 1. The magnetization profiles in molar units as a function of magnetic field for different temperatures. The curves represent interpolated experimental data and the squares with error bars the theoretical estimates. The intercepts report the gaps between linear and parabolic extrapolations when they are convergent.

can be visualized. The uncertainties in the extrapolation decrease with increasing temperature so that the error bars are diminished at $T=10 \mathrm{~K}$. Only a discrete number of data is presented since the Hamiltonian has been diagonalized for each value of the magnetic field and this is the most time consuming task in the numerical calculations.

The relative magnetization $\sigma$ is presented in Fig. 2 as a function of $T / \sqrt{B}$. Results marked by $+, \times, \circ, \bullet, \triangle$, and $\square$ are the theoretical results obtained here. We do not report the experimental results, as they would overlap with ours. The experimental data ${ }^{11}$ turn out to collapse onto a quasiuniversal curve at temperatures above 2

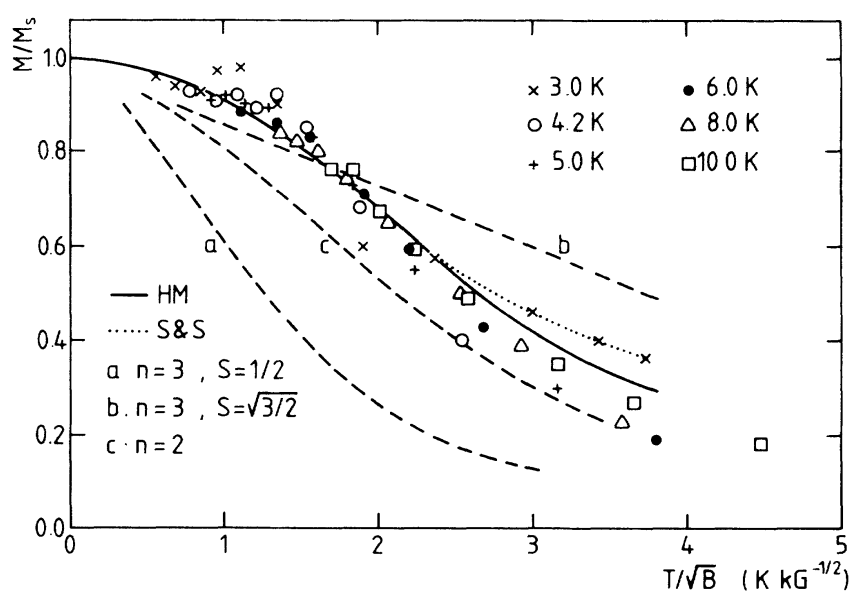

FIG. 2. The relative magnetization as a function of $T / \sqrt{B}$. The $+, \times, \circ, \bullet, \square$, and $\triangle$ denote the present data. The solid line is the universal curve for the model with linear quantum spin-wave excitations and nonlinear classical soliton excitations. The dotted line reports some part of SG predictions and the dashed lines represent the corresponding results for the classical $n$-component model calculated at $T=10 \mathrm{~K}$. 
K. This behavior agrees with the SG predictions: ${ }^{24}$ the relative magnetization $\sigma$ is a universal function of $T / \sqrt{B}$. The experimental findings are represented to some extent by the continuous curve in Fig. 2 . This curve constitutes a good overall representation of the experimental data ${ }^{11}$ and was found from the hybridized model. According to that model, ${ }^{11}$ the thermodynamics of the system (1) is described by free magnons considered on the quantum-mechanical level and by classical solitons. This is only a heuristic model since the solitons are activated in the temperature range (Fig. 5 in Ref. 11) where the magnetization $\sigma$ calculated in the spin-wave approximation is reduced by a factor of 2 with respect to the saturation value. It is not understood why this freemagnon picture is still valid although the spin-wave deviations are no longer small.

It has been realized ${ }^{11}$ that the magnetization data cannot be interpreted on the basis of the classical model. The predictions at $T=10 \mathrm{~K}$ for the classical three- and two-component model (denoted as $n=3$ and $n=2$, respectively) are plotted by the dashed lines in Fig. 2 . They deviate significantly from the experimental values. They also are not universal and show an opposite tendency with respect to that found experimentally (Fig. 4 in Ref. 11). The classical three-component model with imposed value $S=\frac{1}{2}$ was successfully applied ${ }^{21}$ to interpret the inverse correlation length data obtained from the neutron scattering experiment. The same model fails to explain the magnetization data: the values are underestimated by a factor of 2. The SG predictions ${ }^{11,24}$ are depicted only partially in Fig. 2 by the dotted line. At low $T / \sqrt{B}$ they deviate qualitatively ${ }^{11}$ from the experimental behavior.

The hybridized model curve in Fig. 2 constitutes an upper bound on the experimental results for $T / \sqrt{B}>3$ (Fig. 5 in Ref. 11). It is clear that this curve interpolates our data rather well and our estimates collapse onto a quasiuniversal shape for temperatures $T \geq 5 \mathrm{~K}$, where our extrapolations are more reliable. In previous calculations ${ }^{22}$ a satisfactory agreement between the theoretical and experimental data for the correlation length was revealed. This consistent picture will be confirmed by our QMC data reported in the next section.

\section{QUANTUM MONTE CARLO SIMULATIONS}

The finite-temperature QMC method has been applied recently to a number of $1 D$ spin systems. ${ }^{14,17,25-27}$ The isotropic Heisenberg model as well as the $X Y$ model was studied by Cullen and Landau. ${ }^{17}$ Some thermodynamic functions for the anisotropic Hamiltonian (1) were presented by Satija et al. ${ }^{14}$ In this section we evaluate for the model system (1) the magnetization, correlation functions, and the correlation length.

In order to preserve the notation introduced in Ref. 14, we redefine our Hamiltonian (1) in the following way:

$$
\begin{aligned}
\hat{H}= & -2 J \sum_{n=1}^{N}\left(\hat{S}_{n}^{x} \hat{S}_{n+1}^{x}+\Delta \hat{S}_{n}^{y} \hat{S}_{n+1}^{y}+\hat{S}_{n}^{z} \hat{S}_{n+1}^{z}\right) \\
& -\frac{1}{2} h \sum_{n=1}^{N}\left(\hat{S}_{n}^{z}+\hat{S}_{n+1}^{z}\right),
\end{aligned}
$$

where $h=g \mu_{B} B$ and the values of the parameters coincide with these of (2). Periodic boundary conditions are imposed in (5).

The starting point of the QMC method is the generalized Trotter formula ${ }^{15}$ for the exponential of a sum of $k$ noncommuting operators:

$$
\exp \left(\sum_{i=1}^{k} \hat{\theta}_{i}\right)=\lim _{m \rightarrow \infty}\left[\prod_{i=1}^{k} \exp \left(\frac{\hat{\theta}_{i}}{m}\right)\right]^{m}
$$

The integer $m$ is referred to as the "Trotter index." One can apply this formula to the partition function and map our 1D quantum model (5) to a classical 2D system, which can be studied using classical Monte Carlo methods.

The partition function $Z$ is defined by

$$
Z \equiv \sum_{\sigma}\left\langle\sigma\left|e^{-\beta \hat{H}}\right| \sigma\right\rangle,
$$

where the sum runs over all the eigenstates of the spin chain. In this formula the Hamiltonian (5) can be written as the sum of two-body operators:

$$
\hat{H}=\sum_{n=1}^{N} \hat{H}_{n, n+1}=\sum_{n=1}^{N} \hat{H}_{n, n+1}^{o}+\hat{V}_{n, n+1},
$$

where

$$
\begin{aligned}
& \hat{H}_{n, n+1}^{o}=-2 J \hat{S}_{n}^{z} \hat{S}_{n+1}^{z}-\frac{1}{2} h\left(\hat{S}_{n}^{z}+\hat{S}_{n+1}^{z}\right), \\
& \hat{V}_{n, n+1}=-2 J\left(\hat{S}_{n}^{x} \hat{S}_{n+1}^{x}+\Delta \hat{S}_{n}^{y} \hat{S}_{n+1}^{y}\right) .
\end{aligned}
$$

In the checkerboard decomposition ${ }^{14,17}$ (CBD) one splits the Hamiltonian into two parts, $\hat{H}_{\text {odd }}$ and $\hat{H}_{\text {even }}$, containing the two-body operators with odd and even $n$, respectively. The $m$ th-order Trotter approximation to the partition function is then given by

$$
Z_{\mathrm{CBD}}^{(m)}=\operatorname{Tr}\left[\left(\hat{L}_{\text {odd }} \hat{L}_{\text {even }}\right)^{m}\right],
$$

where

$$
\hat{L}_{\text {odd (even) }}=\prod_{n=\text { odd (even) }}\left[\exp \left[-\frac{\beta}{m} \hat{H}_{n, n+1}^{o}\right] \exp \left[-\frac{\beta}{m} \hat{V}_{n, n+1}\right]\right] .
$$

By introducing the complete sets of states $\left|\sigma_{r}\right\rangle=\left|S_{1, r}, S_{2, r} \cdots S_{N, r}\right\rangle(r=1, \ldots, 2 m)$ one gets

$$
Z_{\mathrm{CBD}}^{(m)}=\sum_{\sigma_{1}, \sigma_{2}, \ldots, \sigma_{2 m}}\left\langle\sigma_{1}\left|\hat{L}_{\text {odd }}\right| \sigma_{2}\right\rangle\left\langle\sigma_{2}\left|\hat{L}_{\text {even }}\right| \sigma_{3}\right\rangle \cdots\left\langle\sigma_{2 m}\left|\hat{L}_{\text {even }}\right| \sigma_{1}\right\rangle,
$$

where 


$$
\begin{aligned}
\left\langle\sigma_{r}\left|\hat{L}_{\text {odd (even) })}\right| \sigma_{r+1}\right\rangle & =\prod_{n=\text { odd (even) }}\left\langle S_{n, r} S_{n+1, r}\left|\exp \left(-\frac{\beta}{m} \hat{H}_{n, n+1}^{o}\right) \exp \left(-\frac{\beta}{m} \hat{V}_{n, n+1}\right)\right| S_{n, r+1} S_{n+1, r+1}\right\rangle \\
& \equiv \prod_{n=\text { odd (even) }} e^{-\beta E_{n, r}},
\end{aligned}
$$

and $r$ is odd (even) for $\hat{L}_{\text {odd }}\left(\hat{L}_{\text {even }}\right)$.

In that way we get an expression for a classical partition function for a 2D lattice of size $N \times 2 m$ :

$$
Z_{\mathrm{CBD}}^{(m)}=\sum_{\sigma_{1}, \sigma_{2}, \ldots, \sigma_{2 m}} \prod_{\langle n, r\rangle} e^{-\beta E_{n, r}},
$$

where $\langle n, r\rangle$ indicates that $n$ and $r$ are both odd or both even. Because of the trace in (11), we have periodic boundary conditions in the Trotter direction. The 2D lattice consists of $\mathrm{Nm}$ blocks of four spins ("vertices") arranged as in Fig. 3 .

In the real-space decomposition (RSD) one applies the Trotter formula to the partition function using Eq. (8):

$$
\begin{aligned}
Z_{\mathrm{RSD}}^{(m)} & =\operatorname{Tr}\left(\hat{L}^{m}\right) \\
& =\sum_{\sigma_{1}, \sigma_{3}, \ldots, \sigma_{2 m-1}}\left\langle\sigma_{1}|\hat{L}| \sigma_{3}\right\rangle\left\langle\sigma_{3}|\hat{L}| \sigma_{5}\right\rangle \cdots\left\langle\sigma_{2 m-1}|\hat{L}| \sigma_{1}\right\rangle,
\end{aligned}
$$

where

$$
\hat{L}=\prod_{n=1}^{N} \hat{L}_{n}=\prod_{n=1}^{N} \exp \left(-\frac{\beta}{m} \hat{H}_{n, n+1}^{o}\right) \exp \left(-\frac{\beta}{m} \hat{V}_{n, n+1}\right) .
$$

By introducing complete sets, the expectation value $\left\langle\sigma_{r}|\hat{L}| \sigma_{r+2}\right\rangle$ can be written as

$$
\begin{aligned}
\left\langle\sigma_{r}|\hat{L}| \sigma_{r+2}\right\rangle=\sum_{\sigma_{r+1}} & \left\langle S_{1, r} S_{2, r}\left|\hat{L}_{1}\right| S_{1, r+1} S_{2, r+1}\right\rangle\left\langle S_{2, r+1} S_{3, r}\left|\hat{L}_{2}\right| S_{2, r+2} S_{3, r+1}\right\rangle \cdots \\
& \times\left\langle S_{n, r+1} S_{n+1, r}\left|\hat{L}_{n}\right| S_{n, r+2} S_{n+1, r+1}\right\rangle \cdots \\
& \times\left\langle S_{N-1, r+1} S_{N, r}\left|\hat{L}_{N-1}\right| S_{N-1, r+2} S_{N, r+1}\right\rangle\left\langle S_{N, r+1} S_{1, r+1}\left|\hat{L}_{N}\right| S_{N, r+2} S_{1, r+2}\right\rangle
\end{aligned}
$$

Again we can consider Eq. (17) as defining the partition function of the classical spin- $\frac{1}{2}$ Ising system on the 2D lattice with $\mathrm{Nm}$ vertices arranged as in Fig. 4. As before, periodic boundary conditions are imposed. The quantity

$$
w_{n, r} \equiv e^{-\beta E_{n, r}}
$$

is called the weight of the vertex. The weights are the

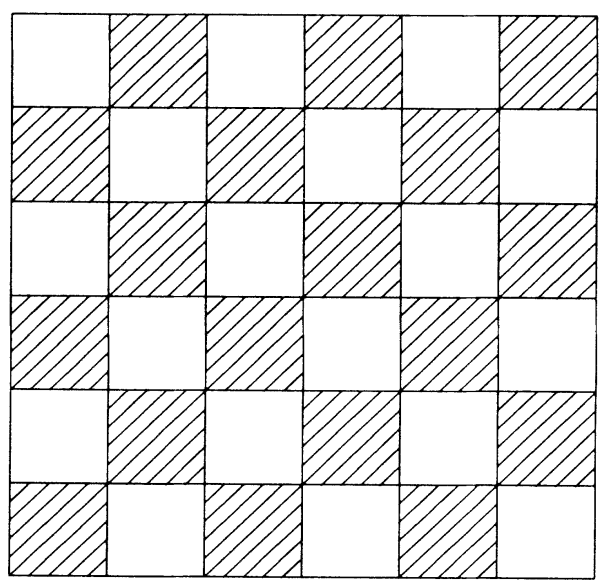

FIG. 3. Arrangement of the vertices in the case of the CBD. The vertices consist of the four spins on the corners of the shaded areas. same for the RSD as for the CBD. They were calculated by Wysin. ${ }^{14,28}$ For the Hamiltonian (5), all vertices with an odd number of spins up have a zero weight and are consequently not allowed, so that we have a so-called eight-vertex model. For the eight-vertex model we can apply the standard Monte Carlo technique ${ }^{14,17}$ (Metropolis algorithm) but the spins at a vertex must always be flipped in pairs. This necessitates flipping a series of spins

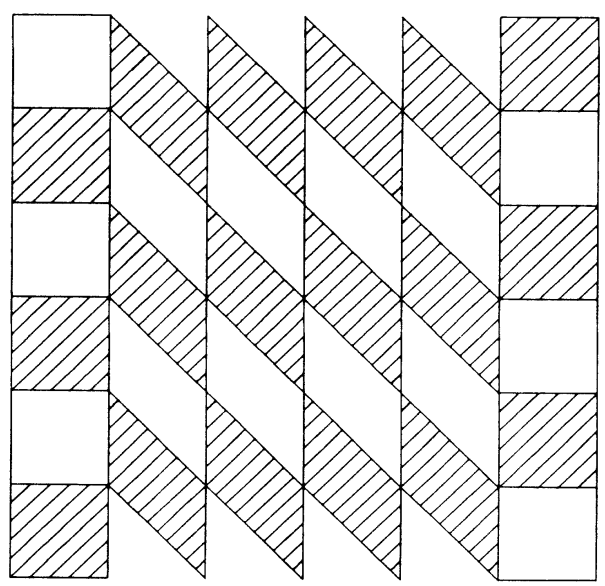

FIG. 4. Arrangement of the vertices in the case of RSD. The vertices consist of the four spins on the corners of the shaded areas. 
in the lattice which lie on a closed path. We have chosen to use only "column flips" (all spins in one column) or "cell flips" (all spins on the corners of a white cell in Figs. 3 and 4). All other allowed spin flips can be obtained by a combination of these. ${ }^{17}$

For CHAB, the thermodynamic quantities we calculated are the magnetization $M_{z}$, the spin-pair correlation function and the inverse correlation length. $M_{z}$ is calculated as the corresponding derivative of the partition function. For both decompositions, in the $m$ th-order Trotter approximation, this gives

$$
\begin{aligned}
M_{z}^{(m)} & =\frac{1}{N} \frac{1}{\beta} \frac{\partial}{\partial h} \ln Z^{(m)} \\
& =\frac{1}{N}\left\langle-\sum_{\langle n, r\rangle} \frac{\partial E_{n, r}}{\partial h}\right\rangle_{\mathrm{cl}} \\
& =\frac{1}{2 m N}\left\langle\sum_{i} S_{i}\right\rangle_{\mathrm{cl}},
\end{aligned}
$$

where the sum over $i$ goes over all spins in the 2D lattice. $\langle\cdots\rangle_{\mathrm{cl}}$ represents the corresponding thermodynamical average for the classical system.

The correlation function,

$$
C_{z z}(k) \equiv \frac{1}{N} \sum_{i=1}^{N}\left\langle\hat{S}_{i}^{z} \hat{S}_{i+k}^{z}\right\rangle,
$$

for the quantum-mechanical system (5) can be calculated from the general expression

$$
\langle\hat{A}\rangle=\frac{1}{Z} \sum_{\sigma}\left\langle\sigma\left|\hat{A} e^{-\beta \hat{H}}\right| \sigma\right\rangle,
$$

which, for all operators $\hat{A}$, can be approximated in the RSD by ${ }^{29}$

$$
\langle\widehat{A}\rangle^{(m)} \equiv \frac{1}{Z^{(m)}} \sum_{\sigma}\left\langle\sigma\left|\hat{A} \hat{L}^{m}\right| \sigma\right\rangle .
$$

For the correlation function this gives

$$
C_{z z}^{(m)}(k)=\frac{1}{N m} \frac{1}{Z^{(m)}} \sum_{K}\left(\sum_{i} \sum_{r \text { odd }} S_{i, r} S_{i+k, r}\right) e^{-\beta E_{K}},
$$

where the $\Sigma_{K}$ goes over all the states of the corresponding classical system and $E_{K}$ is the total energy in that state.

The inverse correlation length $\kappa$ is defined ${ }^{30}$ as

$$
\kappa=\frac{1}{\xi}=\lim _{k \rightarrow \infty} \ln \left(\frac{C_{z z}(k+1)}{C_{z z}(k)}\right) .
$$

In practice, one cannot take the limit, because of the finite size of the system. Moreover, for large $k$, the value of $C_{z z}^{(m)}(k)$ is too small to be calculated accurately by MC. As a compromise, we calculated the inverse correlation length as the average of the logarithm in (27) for $k$ between one and two times the estimated correlation length. We find that, in order to reduce the finite-size effects on the correlation function, one has to use very big systems: five to six times the correlation length is the absolute minimum for the system size $N$. The length $N$ of the system we used lay between 60 , for high temperatures, and 200, for very low temperatures. Some test calculations for chains as long as $N=300$ were also performed to check the absence of finite-length effects.

At this point it is useful to compare the two classical representations of the quantum system (RSD and CBD). In the framework of the quantum transfer matrix method, Betsuyaku ${ }^{18}$ showed that both decompositions give the same results for the thermodynamic properties of the 1D Heisenberg and $X Y$ model in the limit $m \rightarrow \infty$. However, the rate of convergence depends on the choice of the decomposition and RSD is superior to CBD. On the other hand, due to the irregularity of the lattice, the program for the RSD is much more complicated and less efficient than for the CBD. For that reason, we chose the CBD to calculate the magnetization. Our calculations of the correlation function, however, are performed for the RSD. The CBD was found ${ }^{25}$ to destroy the elementary property that the $i$ th spin is to the left of the $(i+1)$ th spin.

The MC calculations were carried out using the Metropolis algorithm. The initial configuration, however, cannot be chosen completely random, because allowed states have an even number of spins up for each vertex. In order to satisfy that condition, we used the following procedure to generate a random allowed state: we started from a uniform state with all spins up, and applied at random $N$ column flips, $N m$ cell flips and again $N$ column flips.

In a single Monte Carlo step (MCS), we attempted $N$ column flips and $\frac{1}{2} \mathrm{Nm}$ square flips, so that, on the average, there are two spin-flip trials per spin per MCS. Data for the expectation values were collected every ten steps. For each value of $T, h$, and $m$, we performed 3-6 MC runs of $(3-5) \times 10^{4} \mathrm{MCS}$ each. These results were averaged and the error bars were determined from their spread.

The method and program have been tested by applying the method to the $X Y$ model $(\Delta=0)$. For this model, some thermodynamic quantities and the correlation length are exactly known. ${ }^{30,31}$ We calculated the correlation function and the correlation length. The results for $\kappa$ are presented in Table I. For $T \geq 15 \mathrm{~K}, \kappa$ was $m$ independent within the statistical error. For these temperatures, the values in Table $I$ are calculated for $m=8$.

TABLE I. Monte Carlo results for the transverse inverse correlation length for the $X Y$ model $\left(\kappa_{\mathrm{MC}}\right)$ in comparison with the exact transverse inverse correlation length $(\kappa)$. The error estimates for $\kappa_{\mathrm{MC}}$ are denoted in brackets.

\begin{tabular}{llll}
\hline$T(\mathrm{~K})$ & $k_{B} T / J$ & $\kappa$ & $\kappa_{\mathrm{MC}}$ \\
\hline 137.5 & 2.5 & 1.639 & $1.65(5)$ \\
110 & 2 & 1.428 & $1.43(5)$ \\
82.5 & 1.5 & 1.167 & $1.18(3)$ \\
55 & 1 & 0.839 & $0.845(8)$ \\
27.5 & 0.5 & 0.428 & $0.430(7)$ \\
15 & 0.273 & 0.236 & $0.215(5)$ \\
10 & 0.182 & 0.158 & $0.150(10)$ \\
5 & 0.091 & 0.079 & $0.065(10)$ \\
\hline \hline
\end{tabular}




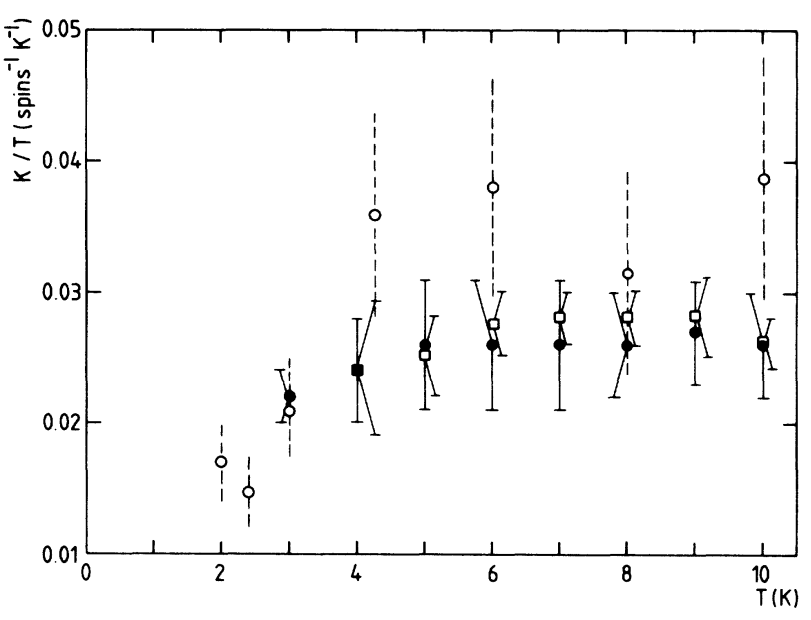

FIG. 5. The inverse correlation length $\kappa / T$ vs temperature for CHAB. The open circles represent the experimental results, solid circles the finite-size results, and the squares represent the MC data. If the error bars overlap, they are drawn to the left for the finite-size results and to the right for the MC results.

For lower temperatures we calculated $\kappa$ for $m=6,7,8$ and extrapolated to infinite $m$, referring to the linear extrapolation in $1 / \mathrm{m}^{2}$. The errors (denoted in parentheses) are estimated from the statistical fluctuations of the results for different runs. As one can see from Table I, the agreement between the MC data and the exact results ${ }^{30,31}$ is very good, except at very-low temperatures.

For CHAB we calculated the correlation function at zero field and temperatures from 4 to $10 \mathrm{~K}$, using the RSD. The results are represented in Fig. 5 by squares, and compared with experimental data ${ }^{21}$ (open circles) and results from finite-size calculations, ${ }^{22}$ denoted by solid circles. Only at $T<6 \mathrm{~K}$ the $m$ dependence was found and the linear extrapolations in $1 / \mathrm{m}^{2}$ were performed.

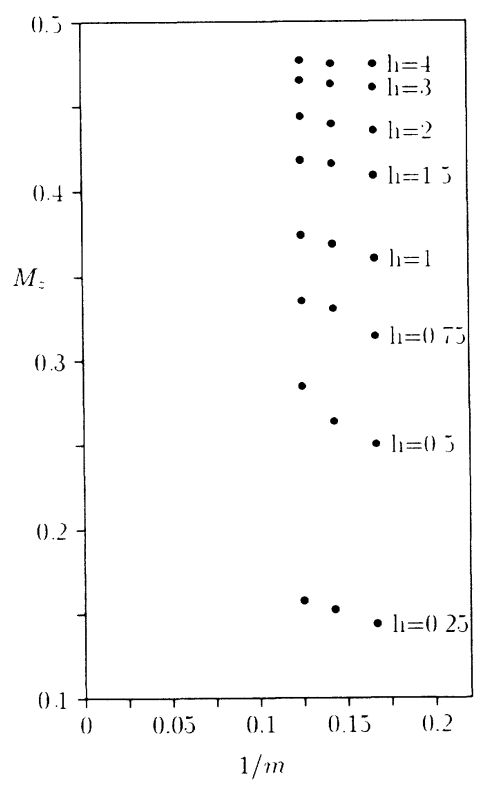

FIG. 6. $1 / \mathrm{m}$ dependence of the magnetization at $T=4.2 \mathrm{~K}$ for different fields.

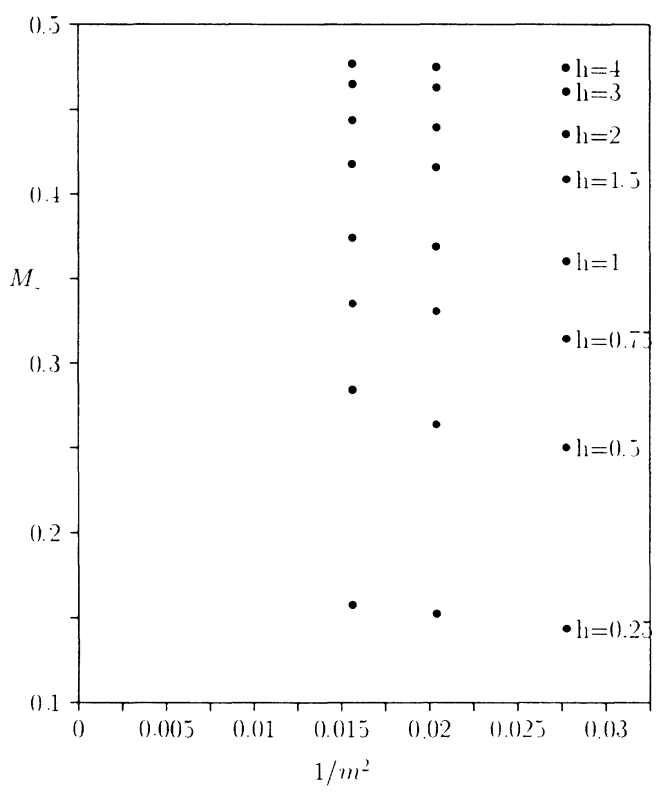

FIG. 7. The same as Fig. 6, but as a function of $1 / \mathrm{m}^{2}$.

For $T \geq 6 \mathrm{~K}$ the results for $m=8$ were reported since they coincide within the statistical errors with the corresponding data for $m=6$ and $m=7$. As one can see, although the MC results agree within the statistical error with the finite-size results the MC data are systematically somewhat higher. However, they remain below the experimental data. We tried to extend the calculations to lower temperature, in order to see clearly the crossover to the $X Y$ behavior in the low-temperature limit, but the MC results started to deviate in the opposite direction. As we considered chains as long as $N=300$ (to avoid finite-size effects), the reason for these deviations might be associated to the length of the 2D system in the Trotter direction, which becomes comparable with the correlation length.

To evaluate the magnetization, we used the checker-

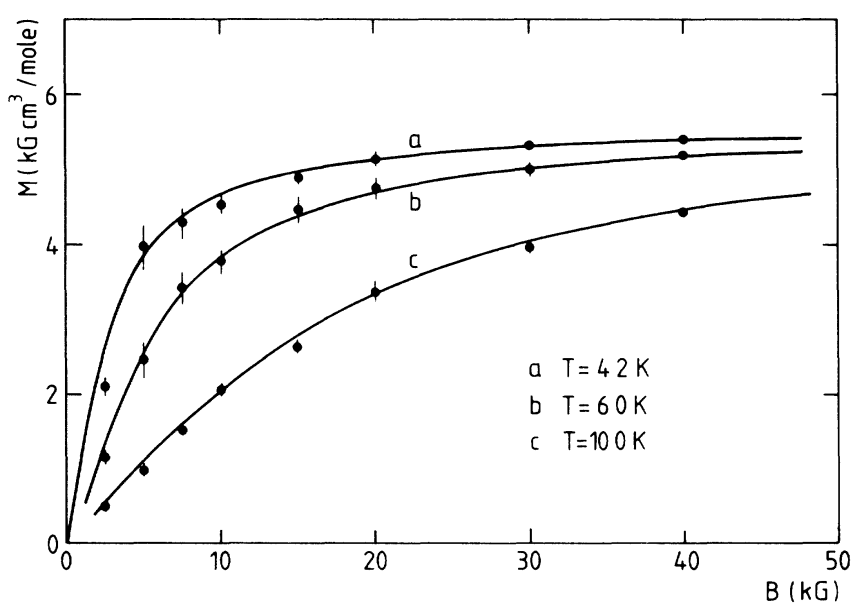

FIG. 8. The MC results for the magnetization of CHAB in molar units, represented by dots. The solid lines represent experimental data. 
board decomposition. The size of the system was $N=100$, which is much more than the correlation length at the considered temperatures $(4.2,6$, and $10 \mathrm{~K})$. The in-plane magnetic field $h$ ranged from 0 to $5 \mathrm{~T}$. We performed the calculations for different Trotter indices: $m=6,7,8$. Because there was a clear $m$ dependence, especially at low $T$ and $h$ (see Figs. 6 and 7), we extrapolated our results for $m \rightarrow \infty$ as a function of $1 / m$ and $1 / m^{2}$. A linear $1 / m^{2}$ dependence is expected for the internal energy. ${ }^{18}$ In our calculation of $M_{z}$, we do not recover the perfect linear $1 / \mathrm{m}^{2}$ dependence, so that we perform the linear extrapolation in $1 / \mathrm{m}$, too. A parabolic extrapolation could not be used because of the small number of data points and the sensitivity to small shifts of the data. Our final results for the molar magnetization (8) are the averages of these two extrapolations and are represented in Fig. 8 by solid circles. There is an excellent agreement between our MC data and the experimental results, represented by the continuous lines. As the estimates of the error bars, we assume the difference between these two extrapolations. The data for a given $m$, $T$, and $h$ are subject to statistical fluctuations. The standard deviations do not exceed $10 \%$ at low fields and systematically decrease down to $1 \%$ at higher fields.

With these simulations we have shown that the quantum Monte Carlo method, based on the Trotter formula, is a valuable tool to study one-dimensional quantum chains. We have also confirmed the finite-size results as presented in Sec. 2 and Ref. 22. In the near future, we want to apply this method to calculations of other thermodynamic quantities, so that further comparison between the quantum model (1) and the experimental data for $\mathrm{CHAB}$ will be possible.

\section{CONCLUDING REMARKS}

We have presented the numerical results for the zerofield correlation length and the field dependent in-plane magnetization for the anisotropic spin- $\frac{1}{2}$ easy-plane ferromagnetic Heisenberg model (1), as well as making contact with the corresponding experimental data on CHAB. Two types of theoretical analysis have been put forward: the finite-size extrapolations in $1 / N$ and the quantum Monte Carlo simulations based on the Trotter formula. The latter confirm very well the present finite-size estimates of the magnetization and previous ${ }^{22}$ estimates of the correlation length.

In view of some difficulties inherent to the QMC method, we emphasize that some standard precautions have been taken into account. The length of the chains we have considered (ranging from $N=60$ up to $N=300$ ), was at least a factor of 10 larger than the correlation length. The length of the Monte Carlo runs was of the order $5 \times 10^{4}$, and we performed 3-6 MC runs in order to carry out the statistical analysis. The heating procedures preventing correlations between states were also imposed. Test calculations of the correlation length for the spinpair correlation function of the $X Y$ model have been performed and excellent agreement with the exact results ${ }^{30,31}$ has been found.

Clearly, no direct information about the excitations has been gained and the existence of solitons has not been established. However, the importance of quantum effects has been indicated and the validity of the Hamiltonian (1) as an adequate model for CHAB strongly supported. For the first time, rather good agreement with the experiment has been revealed for the quantities in question. Immediately, the problem of some inconsistencies, existing in the interpretation of the in-plane specific-heat data, ${ }^{20,23}$ is raised. We expect that the present status constitutes an appeal for new refined experimental data for the specificheat on $\mathrm{CHAB}$.

\section{ACKNOWLEDGMENTS}

We would like to thank Professor W. J. M. de Jonge and Dr. K. Kopinga for providing us with their experimental data before publication as well as many clarifying discussions on CHAB, Professor V. Tognetti for presenting to us the status of the sine-Gordon theory, $K$. Binder for helpful discussions. G.K. would like to thank the Institute of Physics of the Lodz University for the partial support via the project CPBP 01.08.
*Permanent address: Instytut Fizyki, Uniwersytet im A. Mickiewicza, 60-769 Poznan, Poland.

${ }^{1}$ M. Steiner, J. Villain, and G. G. Windsor, Adv. Phys. 25, 87 (1976); M. Steiner, in Physics in One Dimension, edited by J. Bernasconi and T. Schneider (Springer-Verlag, Heidelberg, 1981).

${ }^{2}$ Magnetic Excitations and Fluctuations, edited by S. W. Lovesey, U. Balucani, F. Borsa, and V. Tognetti (SpringerVerlag, Heidelberg, 1984).

${ }^{3}$ Magnetic Excitations and Fluctuations II, edited by U. Balucani, S. W. Lovesey, M. G. Rasetti, and V. Tognetti (Springer-Verlag, Heidelberg, 1987).

${ }^{4}$ M. Steiner, K. Kakurai, and J. K. Kjems, Z. Phys. B 53, 117 (1983).

${ }^{5}$ K. Kopinga, A. M. C. Tinus, and W. J. M. de Jonge, Phys. Rev. B 25, 4685 (1982)

${ }^{6}$ K. Kopinga, A. M. C. Tinus, and W. J. M. de Jonge, Phys.
Rev. B 29, 2868 (1984).

${ }^{7}$ H. A. Groenendijk, H. W. J. Blöte, A. J. van Duynenveldt, R. M. Gaura, C. P. Landee, and R. D. Willet, Physica (Utrecht) 106B, 47 (1981).

${ }^{8}$ C. P. Landee, A. C. Lamas, R. E. Greeny, and K. G. Bücher, Phys. Rev. B 35, 228 (1987).

${ }^{9}$ P. M. Duxbury, J. Oitmaa, M. N. Barber, A. van der Bilt, K. O. Joung, and R. L. Carlin, Phys. Rev. B 24, 5149 (1981).

${ }^{10}$ A. P. Ramirez and W. P. Wolf, Phys. Rev. Lett. 49, 227 (1982); Phys. Rev. B 32, 1639 (1985).

${ }^{11}$ K. Kopinga, A. M. C. Tinus, W. J. M. de Jonge, and G. C. de Vries, Phys. Rev. B 36, 5398 (1987).

${ }^{12}$ E. Magyari and H. Thomas, J. Phys. C 15, L333 (1982).

${ }^{13}$ G. Kamieniarz and C. Vanderzande, Phys. Rev. B 35, 3341 (1987).

${ }^{14}$ I. Satija, G. Wysin, and A. R. Bishop, Phys. Rev. B 31, 3205 (1985); G. M. Wysin and A. R. Bishop, ibid. 34, 3377 (1986). 
${ }^{15}$ M. Suzuki, Prog. Theor. Phys. 56, 1454 (1976).

${ }^{16}$ J. C. Bonner and M. E. Fisher, Phys. Rev. A 135, 640 (1964).

${ }^{17}$ J. J. Cullen and D. P. Landau, Phys. Rev. B 27, 297 (1983).

${ }^{18}$ H. Betsuyaku, Prog. Theor. Phys. 73, 319 (1985).

${ }^{19}$ A. C. Phaff, C. H. W. Swüste, W. J. M. de Jonge, R. Hoogerbeets, and A. J. van Duynevelt, J. Phys. C 17, 2583 (1984).

${ }^{20}$ A. M. C. Tinus, W. J. M. de Jonge, and K. Kopinga, Phys. Rev. B 32, 3154 (1985).

${ }^{21}$ K. Kopinga, W. J. M. de Jonge, M. Steiner, G. C. de Vries, and E. Frikkee, Phys. Rev. B 34, 4826 (1986).

${ }^{22}$ G. Kamieniarz, Phys. Rev. B (to be published).

${ }^{23}$ G. Kamieniarz, Solid State Commun. (to be published).

${ }^{24}$ T. Schneider and E. Stoll, Phys. Rev. B 22, 5317 (1980).
${ }^{25}$ H. De Raedt, A. Lagendijk, and J. Fivez, Z. Phys. B 46, 261 (1982).

${ }^{26} \mathrm{H}$. De Raedt, in Magnetic Excitations and Fluctuations II, edited by U. Balucani, S. W. Lovesey, M. G. Rasetti, and V. Tognetti (Springer-Verlag, Heidelberg, 1987), p. 204.

${ }^{27}$ M. P. Nightingale and H. W. J. Blöte, Phys. Rev. B 33, 659 (1986).

${ }^{28}$ G. Wysin, Ph.D. thesis, Cornell University, Ithaca, NY (unpublished).

${ }^{29}$ H. De Raedt and A. Lagendijk, Phys. Rep. 127, 233 (1985).

${ }^{30}$ T. Tonegawa, Solid State Commun. 40, 983 (1981).

${ }^{31}$ S. Katsura, Phys. Rev. 127, 1508 (1962). 